\title{
Intra-annual to multi-decadal xylem traits in a tropical moist semi-deciduous forest of Central Africa'
}

\author{
Tom De Mil \\ Department of Forest and Water Management, Laboratory of Wood Technology, \\ Faculty of Bioscience Engineering, Ghent University \& Royal Museum for Central \\ Africa, Wood Biology Service
}

\begin{abstract}
A witness of a tree's past conditions is the wood itself. The main research question of this dissertation is how to assess and reveal the driving forces of the patterns of wood traits on pith-to-bark cross-sections in tropical trees. Cambial and leaf phenology was monitored in the Luki Reserve (Mayombe forest, D.R. Congo). Furthermore, X-ray CT densitometry was explored to assess traits in a reliable way, for multiple species. Variability in phenology is observed for T. superba, which requires traits of individual trees to be fixed on a time axis. Furthermore, $\mathrm{X}$-ray CT is a suitable method for assessing traits in a fast way. Cambial activity of understory trees has shown to be species-specific, whilst many trees show zero xylem growth. Finally, 66 years of tree growth was analysed, while trait analysis revealed a median ring count of only 32 , thus implying many nonperiodical rings. This work presents methodological improvements to measure traits as continuous variables from pith to bark, but also acknowledges that phenology still remains a key aspect in order to fix traits on a time axis.

Key words: leaf phenology, wood anatomy, stable isotopes, Mayombe, dendrochronology, X-ray CT densitometry
\end{abstract}

Tropical forests cover a large patch of land on Earth's surface. Forests are disappearing or changing in structure due to increased human pressure. Furthermore, current forests are prone to climatic changes, and as a major sink for carbon, they have a vital role in Earth's carbon balance. Given the fact that these forests are pushed into an unprecedented environment, quantifying and predicting the evolution of tropical forests to future climate change is urgent. The trunk contributes to the largest part of tree biomass. Biomass gain in the trunk occurs through the formation of irreversible growth layers of xylem (wood), which causes radial growth. Live monitoring of radial growth occurs through permanent sample plots, though they do not reach further than a few decades and detailed knowledge on xylem growth and carbon gain cannot be assessed.

This is the report of PhD research carried out at Ghent University, under the supervision of Joris Van Acker, Jan Van den Bulcke and Hans Beeckman. 
A key witness of the tree's past conditions is the xylem archive. In extratropical trees, the alteration of winter and summer induces alterations of xylem growth followed by dormancy, which forms clear visual rings in the xylem. Tree-ring analysis explores the xylem archive through detailed counting, measuring and synchronizing (i.e. cross-dating) ring widths in the xylem and allows to estimate tree age, assess historic tree response, and reconstruct past climate conditions. In tropical trees, many trees form rings in the xylem but these are more subtle and mainly caused by alternation of dry and rainy conditions. Tree-ring analysis has been shown successful for matching ring-width sequences in tropical trees, revealing long-term growth patterns and age of tropical trees. However, originally designed for extratropical trees, many ring-width based dendrochronological studies in tropical trees report difficulties in cross-dating, which results in discarding many samples, and causing large uncertainties, with many species being ignored. There is, however, more than only ring width to take into account. Looking between the ring boundaries, intra-annual xylem traits are of chemical and structural nature and provide us with seasonal information of tree response. In order to have a mechanistic understanding of when these traits are formed, knowledge of recurring events of meristems is essential: leaf phenology (buds) and cambial phenology (vascular cambium). Xylem traits from pith to bark are increasingly being explored to map tree performance in the past. In tropical trees, linking xylem structure to the past is less evident and depends on a thorough assessment of leaf and cambial phenology.

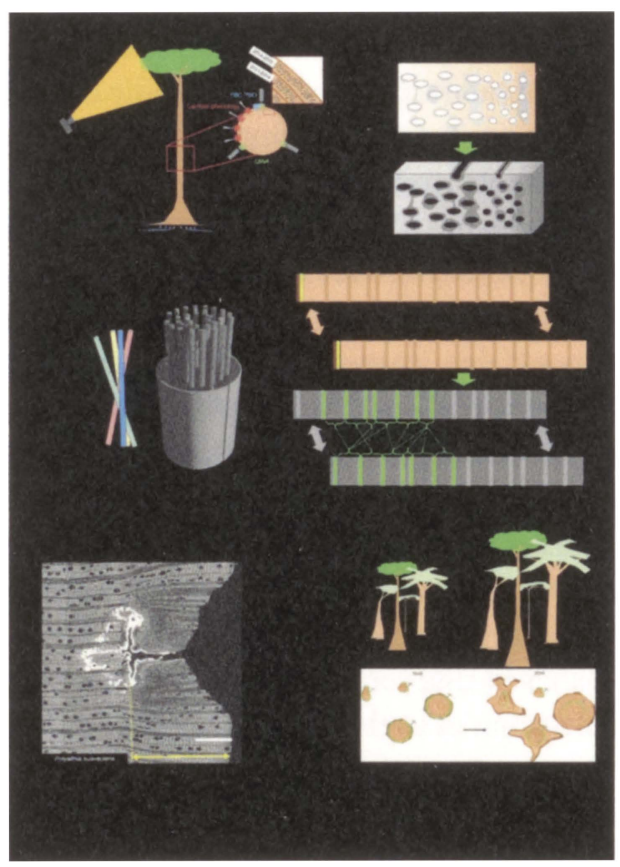

Figure 1: Thesis outline. 
Thus, this dissertation aims at exploring the xylem archive beyond the assessment of tree ring boundaries in traditional tree-ring analysis. We investigate intra-annual to multi-decadal xylem traits on tree species from the Luki UNESCO Man And Biosphere Reserve, which is part of a semi-deciduous tropical rainforest at the southernmost edge of the Mayombe forest near the Atlantic Ocean (western DR Congo). This study site is of special interest due to the presence of inter- and intra-species variation in leaf and cambial phenology.

Firstly, we focus on Terminalia superba, a tree species with a known dendrochronological potential, but with large variations in leaf phenology and a considerable variation in intra-annual xylem traits such as density fluctuations between the tree ring boundaries. During two growing seasons, leaf phenology, daily stem diameter variations, and cambial phenology were monitored to disentangle tree response. Leaf onset differences up to 45 days were observed between trees and persisted between years, irrespective of rainfall onset, resulting in tree-specific xylem onsets. The rings that were formed during monitoring were subjected to intra-annual wood cellulose isotope and wood anatomical measurements. Differences in cambial phenology result in tree-specific timeframes of growth, and the resulting intra-annual traits should be compared between trees on an intra-annual time axis, rather than a distance axis within an inter-annual timeframe (Fig. I) (Chapter 2).

Secondly, X-ray Computed Tomography (X-ray CT) microdensitometry is proposed as a technique to assess intra-annual traits in a uniform and fast way. In tropical angiosperm tree species such as T. superba, the presence of fibres, parenchyma and vessels complicates the interpretation of the density profile. Therefore, a decomposition of intra-annual profiles into its anatomical components was necessary and showed that vessels and parenchyma decrease wood density, whereas fibre wall fractions, and proportion positively affect wood density. The slowly increasing fibre wall fraction along the development of the tree ring abruptly ends and delineates the tree ring boundary. $\mathrm{X}$-ray CT microdensitometry is not only an appropriate method to define tree ring boundaries, but the resulting intra-annual density profiles were proven to be directly linked to intraannual wood traits in T. superba (Fig. I) (Chapter 3).

Thirdly, to upscale the technique, we present an X-ray CT microdensitometry tool chain of tree increment cores, resulting in profile datasets suitable for visual exploration as well as density-based pattern matching. A digital workflow allows to generate density profiles of large sets of cores in a short time span that provide sufficient intraannual density information for tree-ring analysis. Density-based pattern matching is able to formally detect anomalies in ring series that can be corrected via interactive software. Furthermore, visual exploration of such datasets is of high value, as patterns emerge of trees that have similar intra-annual information in their rings. The dated profiles can be used for high-resolution chronologies and also offer opportunities for fast screening of lesser studied tropical tree species (Fig. I) (Chapter 4).

Subsequently, we expanded the above methods to multiple species of the Mayombe, both understory and canopy species. Knowledge on intra-annual xylem growth remains 
understudied in tropical regions, especially for understory species. The cambium of 4 species was monthly marked at the stem base via the pinning method. In many cases, intra-annual growth was successfully derived and could be fitted with a Gompertz function. A species-specific response is observed, which is assumed to be due to phenology differences, but variation between trees of the same species is observed as well. Anomalies such as circumferential variability and wedging were reported, but the most remarkable result is that many of the trees in the dataset had no xylem formation at the stem base, throughout the entire season. Intra-annual variability in growth illustrates the different responses of species and individual trees to environmental drivers. Leaf phenology might explain the differences, although site and influence of other trees should be considered as well. A large number of trees show no xylem growth at all, apart from wound-induced local growth (Fig. I) (Chapter 5).

Going from the intra-annual level to the multi-decadal level, xylem traits were assessed by the use of a unique set of rediscovered trees from the Nkulapark, an old forest growth and phenology monitoring park. Records of yearly diameter data of 4767 trees covering 176 species measured from 1948 to 1957 , were digitized and analysed, and compared to $4 \mathrm{I} 4$ trees that were rediscovered in 2014. The diameter of the rediscovered trees was measured and the nails carrying the original 1948 number tag serve as a cambial timestamp in the wood, an unprecedented opportunity to directly assess wood formation. 134 samples from 18 species were taken, containing the nail marks in order to analyse radial xylem growth. The average radial increment over the 66-year timespan was highly variable: some understory trees showed no radial increment at all, while other canopy species showed up to $30 \mathrm{~cm}$ radial growth. When the xylem structure was examined, ring structures were visible on all species but it was shown that in some cases only few discernible rings were formed in understory trees. Some canopy trees showed considerable radial growth, had rings that concur with the calendar years elapsed and thus give a reliable view on the tree's past. Additionally, canopy tree species were found with radial growth, but the number of rings did not concur with the years elapsed. The low increment of understory species, can also be a reason that the oldest tropical tree should not only be sought among the tallest trees. Anomalies were thus detected on the intraannual scale, and confirmed at the multi-decadal scale (Fig. I) (Chapter 6).

In the last section of this dissertation, a discussion is held to reconsider these anomalies as an opportunity for trait measurements on a wide range of tropical trees. Instead of discarding samples with irregular or missing rings, they should be further investigated via xylem traits from an equatorial viewpoint, rather than applying tree-ring analysis on a limited set of species (Fig. I) (Chapter 7).

The main outcomes of this dissertation are:

- Evidencing that within a tropical species with a known dendrochronological potential such as T. superba, phenology differences lead to considerable shifts in xylem traits along a time axis.

- Decomposing the intra-annual density profile into its xylem anatomical components shows that $\mathrm{X}$-ray $\mathrm{CT}$ densitometry allows to assess intra-annual traits. 
- Developing an X-ray CT densitometry tool chain while developing a formal method to synchronize density profiles based on density information.

- Demonstrating that several species in the Mayombe show distinct tree ring boundaries, but the number of discernible rings seldom matches the number of elapsed years. Moreover, some trees appeared to have no xylem growth at the stem base after 66 years.

Traditional tropical tree-ring analysis can only be performed on a limited set of species, while permanent sample plots do provide the ecological integration of all species, but can only be assessed in periodical terms. However, Xylem traits, whether or not measured via X-ray CT densitometry, are scaled from the intra-annual to the multidecadal level and should therefore be further explored to evaluate carbon stocks through time in tropical forests.

\section{References}

De Mil, T. (2017). Intra-annual to multi-decadal xylem traits in a tropical moist semi-deciduous forest of Central Africa. PhD Thesis, Ghent University, Belgium. 